\title{
Similarity space for auditory signals differing in frequency and intensity
}

\author{
IRWIN POLLACK and NAIF KHOURI \\ Mental Health Research Institute, University of Michigan, Ann Arbor, Michigan 48109
}

\begin{abstract}
Zagorski (1973) found that listeners appear to ignore the value of a fixed variable when adjusting the value of another variable in equisimilarity matching to tones differing with respect to two variables. We explore the robustness of this finding and explore the form of instructions required for the listeners' adjustments to be sensitive to both variables.
\end{abstract}

When subjects are asked to judge the similarity among different multidimensional stimuli, their judgments often reflect the separate dimensions over which the stimuli are varied. Some classes of stimuli appear to yield a similarity space that is most consistent with a city-block solution; some classes appear to yield a euclidean solution. Indeed, Garner (1974) and Shepard (1964) have been able to categorize the stimulus situation as "analyzable" or "separable," or "nonanalyzable" or "integral," in terms of the form of the similarity solution. Regardless of the form of the solution, the ratings appear to reflect both variables.

However, when subjects are given control of one variable, Zagorski (1973) finds that subjects ignore the other variable in making adjustments for equisimilarity. Typically, Zagorski emphasizes speed of adjustment and, through speed stress, he may have forced his listeners into a one-dimensional solution. The present tests explore the instructional set required to overcome the "one-dimensional" solution in an adjustment task.

\section{SECTION 1: INSTRUCTION AND PRODUCTION SERIES}

\section{Method}

Five university students, each with instrumental music experience, but without psychophysical experience, were tested individually. The listener controlled a five-position switch marked "A, silence, B, silence, C," which permitted presentation of Tones $A, B$, and $C$ with interspersed silent periods. The listener also controlled either an oscillator or an attenuator. The oscillator varied the frequency of Tone $B$; the attenuator varied the intensity of Tone B. The listener's task was to adjust the oscillator or the attenuator so that Tone B would be equally similar to Tones $A$ and $C$. Tones $A$ and $C$ were fixed in intensity and frequency by the experimenter (Tone $A$ at $1,500 \mathrm{~Hz}$, $60 \mathrm{~dB}$ SPL, Tone $\mathrm{C}$ at $800 \mathrm{~Hz}, 80 \mathrm{~dB}$ SPL). Each subject made eight successive intensity (frequency) adjustments followed by eight successive frequency (intensity) adjustments. No emphasis was placed on speed of adjustment. The order of adjustment within a variable, and across variables, was scrambled within and across listeners. Each set of adjustments was made following one of the graded series of instructions or productions, shown in Table 1. The standard equal-similarity instruction was Stage 1.

Requests for reprints should be sent to the first author. This research was aided in part from a grant by the National Sciences Foundation.
Table 1

Stages of Instruction or Production

\section{Instruction Series}

1. Adjust Tone B so that its perceived similarity is equidistant between Tones $A$ and $C$. In terms of similarity, you want to adjust $B$ so that the similarity between $A$ and $B$ is equal to the similarity between $B$ and $C$.

2. Stage 1 , plus noting that Tones $A$ and $C$ differ in both pitch and loudness and that you will be controlling the pitch (or the loudness) while the experimenter controls the other variable.

3. Stages 1 plus 2 plus the statement that an increase in pitch must be offset by an increase in loudness.

4. Subject is shown a two-dimensional map of the space of possible tones with $\mathrm{A}$ and $\mathrm{C}$ indicated as two diagonal corners of a square with axes marked pitch and loudness. Subject was asked to indicate the locus of positions equidistant to $A$ and $C$ in the map space. The map was then removed and Stage 1 instructions were repeated.

5. Stage 4 except that the subject was instructed to use the map as the similarity space in making final adjustments under Stage 1 instructions.

\section{Production Series}

11. Experimenter presents Tone B. Subject plots Tone B with a point upon a two-dimensional grid with the axes marked pitch and loudness, and with endpoints $A$ and $C$ spaced $16.5 \mathrm{~cm}$ apart vertically and horizontally. Map is then removed.

12. Subject is shown a two-dimensional map of the space of possible tones with $A$ and $C$ marked. Subject adjusts both the intensity and frequency of Tone $B$ to match marked positions upon the grid.

13. Both Tones $\mathrm{A}$ and $\mathrm{C}$ are varied by the experimenter between successive adjustments; the subject adjusts Tone B to equisimilarity.

Stage 2 pointed out that Tones $\mathrm{A}$ and $\mathrm{C}$ differed with respect to two variables and that the subject controlled only one of the variables. Stage 3 provided a specific statement for the twodimensional solution. Stage 4 suggested a two-dimensional space for plotting Tones $\mathrm{A}$ and $\mathrm{C}$ and showed the locus of equidistant points. Stage 5 extended Stage 4 in that the subject employed the two-dimensional plot in making equisimilarity judgments.

The order of testing was as follows: Subject 1-1 $(11,12), 5$; Subject 2-1 $(11,12), 5$; Subject 3-2, 4, 5; Subject 4-1, 13, 5; Subject $5-3,12,5$. The rise-fall time of each tone was $2.5 \mathrm{msec}$.

\section{Results}

The test results of a typical listener, Subject 1 , are shown in Figure 1. The left panel displays his individual 


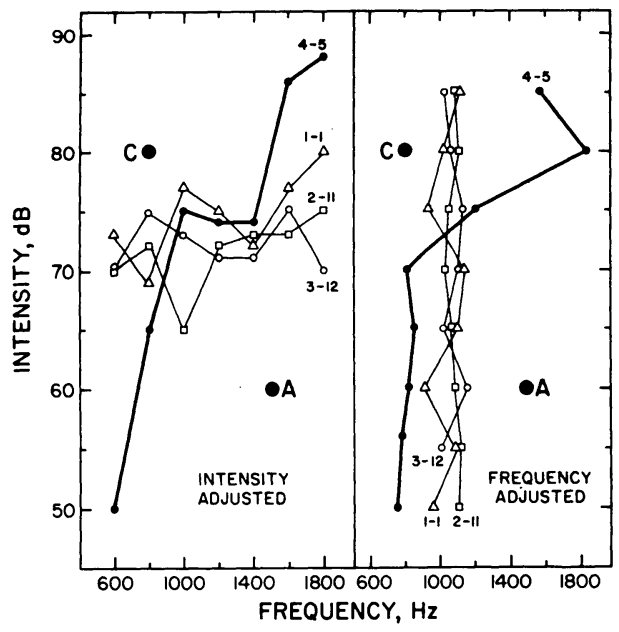

Figure 1. Intensity adjustments (left panel) and frequency adjustments (right panel) of Tone $B$ to equal similarity between Tones $A$ and $C$ when the other variable is controlled by the experimenter. The label on the curves is the order of testing and the instruction or production stage preceding the test.

adjustments in frequency when the intensity was set by the experimenter. The parameter on the curves is the order of the test, followed by the instructional or production task employed. With the exception of performance following Stage 5 , the intensity adjustments for Tone B in the left panel are relatively insensitive to the frequency of Tone $B$, and the frequency adjustments for Tone B ir the right panel are relatively insensitive to the intensity of Tone B. Moreover, the mean adjusted level approaches the mean level of the adjusted variable of Tones $\mathrm{A}$ and $\mathrm{C}$.

Only after the most explicit directed instruction, with the solution space as a visual guide (Stage 5), did this subject reflect a two-dimensional solution to the similarity task. Even under this instruction, his responses were hesitant, with frequent back-and-forth inspections of the map. In the earlier production Test 11, his plotting responses reflected both the frequency and intensity of the tone. In the earlier production Test 12 , his adjustments reflected variations in points along both the frequency and intensity axes. Therefore, one might surmise that the two-dimensional mapping was well understood with respect to frequency and intensity. But with only a single variable to manipulate, specific directed guidance was required to position the tone along the locus of equidistance to $A$ and $\mathrm{C}$ in the two-dimensional space.

All these considerations suggest that the tradeoff between the variables was not appreciated in the context of manipulation of a single variable at a time. This result is obtained despite the indications from the production tests that the separate variables were quickly appreciated.

The results of each subject are summarized in Table 2 in terms of the relative slope of the adjustments with respect to the nonadjusted variable. If the similarity space was actually represented by a decibel/linearfrequency tradeoff, the hypothetical slope would be $20 \mathrm{~dB} / 700 \mathrm{~Hz}$ or $700 \mathrm{~Hz} / 20 \mathrm{~dB}$. Such a hypothetical listener would yield a relative slope of 1.0. The proportion of the total variance accounted by the least-squares regression is also presented. Table 2 is organized by the order of testing and by listener number. For Listeners 1 and 2, results were averaged following two production tests at the intermediate stage.

The general pattern that emerges for each listener is a weak relation between the adjustment and the nonadjusted variable, except following Stage 5 instructions.

Subject 6 represents a subject who was first given a multidimensional rating test (next section). She balked that the task was impossible. She was then shown a map and the tradeoff was explained. Her first adjustments were made with the map available. She then plotted points on the map to unknown sounds. She then repeated the adjustments without the map.

\section{Discussion}

If we employ Zagorski's (1973) criterion of a rapid response, made without hesitation and without inspection of external aids, none of the six listeners demonstrated a two-dimensional solution to the equisimilarity adjustment task. Interestingly, the plotting of an unknown sound in a two-dimensional space marked pitch and loudness was considered easy and was accomplished quickly, as was the adjustment of two variables to match points in two-dimensional space. The adjustment of a single variable, however, required deliberate, effortful visual guidance from the two-dimensional plot.

Table 2

Relative Slope Coefficients (C) and Proportion of Accountable Variance for the Production-Adjustment Series

\begin{tabular}{|c|c|c|c|c|}
\hline \multirow[b]{2}{*}{ Subject } & \multicolumn{2}{|c|}{ Vary Intensity } & \multicolumn{2}{|c|}{ Vary Frequency } \\
\hline & $\mathrm{C}$ & $\mathbf{R}^{2}$ & $\mathrm{C}$ & $\mathrm{R}^{2}$ \\
\hline \multicolumn{5}{|c|}{ Initial Order } \\
\hline 1 & .17 & .33 & .05 & .06 \\
\hline 2 & .24 & $.64^{*}$ & -.01 & .02 \\
\hline 3 & .06 & .06 & -.29 & $.62 *$ \\
\hline 4 & .09 & .42 & .03 & $.66^{*}$ \\
\hline 5 & .27 & $.83 * *$ & .03 & .13 \\
\hline \multicolumn{5}{|c|}{ Intermediate Order } \\
\hline 1 & .07 & .11 & .00 & .02 \\
\hline 2 & .18 & .54 & .00 & .06 \\
\hline 3 & .08 & 14 & .03 & .12 \\
\hline 4 & .03 & .06 & .06 & .46 \\
\hline 5 & .12 & .32 & .09 & .02 \\
\hline \multicolumn{5}{|c|}{ Final Order } \\
\hline 1 & .98 & $.87 \dagger$ & .82 & $.72 * *$ \\
\hline 2 & 1.02 & $.88 t$ & .79 & $.96+t$ \\
\hline 3 & .80 & $.90 \dagger$ & .91 & $.96+t$ \\
\hline 4 & .73 & $.91 \dagger$ & .74 & $.86 \dagger$ \\
\hline 5 & 1.02 & $.93 \dagger \dagger$ & .75 & $.91 \dagger$ \\
\hline 6무 & .78 & $.91 \dagger$ & .83 & $.84 \dagger$ \\
\hline 6古蛒 & .67 & $.93 \dagger \dagger$ & .80 & $.89 \dagger$ \\
\hline
\end{tabular}

$\begin{array}{ccc}* p<.05 & t p<.001 & \text { wwith map } \\ * * p<.01 & t \neq p<.0001 & \text { sw without map }\end{array}$




\section{SECTION 2: RATING TESTS}

In the tests of Section 1, the only method that led to two-dimensional equisimilarity adjustments was a directed procedure in which the listeners apparently had little confidence. We felt we should be able to obtain the desired result by less clumsy means.

\section{Method}

Five additional listeners performed three sets of eight ratings each. One aspect of Tone B (e.g., its intensity) was manipulated by the experimenter, while the other aspect (e.g., its frequency) was equal to that of Tone $A$ or Tone $C$. The listener judged the loudness of Tone B on a scale of -100 (identical to the loudness of Tone A) to +100 (identical to the loudness of Tone $\mathrm{C}$ ). The first rating was made with both variables of Tone B set identically to Tone $A$ or to Tone $C$. The second rating was made at the frequency of Tone $\mathrm{A}$ or $\mathrm{C}$ and at the intensity of Tone C or A, respectively.

The second set of ratings manipulated the other aspect of Tone B. In the example given, the intensity of Tone B was first manipulated and the frequency of Tone $B$ was next manipulated. The variables were counterbalanced across listeners.

The third set of ratings manipulated both variables. The listener was required to give separate ratings with respect to both the pitch and the loudness of Tone B, relative to Tones A and C. Following each set of ratings, a single adjustment was required with respect to the manipulated variable(s) of the previous set of ratings. Following the three sets of ratings, the listener was instructed to vary the oscillator or the attenuator so that "the sum of the loudness difference plus the pitch difference between Tones A and B was equal to that between Tones B and C." This was not understandable to all subjects, so that numeric examples were constructed and discussed. After completion of eight adjustments with respect to one variable, the subject completed eight adjustments with respect to the other variable.

\section{RESULTS AND DISCUSSION}

Table 3 presents the relative slope coefficients and the proportion of accountable variance for the final
Table 3

Relative Slope Coefficients (C) and Proportion of Accountable Variance for the Rating-Adjustment Series (Final Order)

\begin{tabular}{cccccc}
\hline & \multicolumn{2}{c}{ Vary Intensity } & & \multicolumn{2}{c}{ Vary Frequency } \\
\cline { 2 - 3 } \cline { 5 - 6 } Subject & $\mathrm{C}$ & $\mathrm{R}^{2}$ & & $\mathrm{C}$ & $\mathrm{R}^{2}$ \\
\hline 11 & .57 & $.67^{*}$ & .44 & $.66^{*}$ \\
12 & .37 & .45 & .77 & $.90^{* *}$ \\
13 & 1.20 & $.88^{* *}$ & .86 & $.76^{*}$ \\
14 & .33 & $.70^{*}$ & .06 & .07 \\
15 & .65 & $.85^{*}$ & .66 & $.72^{*}$ \\
\hline
\end{tabular}

${ }^{*} p<.01 \quad{ }^{* *} p<.001$

set of adjustments. We were only partially successful in developing the two-dimensional solution. Each listener apparently manipulated at least one variable in conjunction with modifications in the other variable in response to the directed solution. It is the experimenter's impression that the solution was less coerced than the visual guidance provided by the two-dimensional plot.

In summary, for tones differing with respect to both frequency and intensity, the equisimilarity solution achieved by adjustment of one variable typically fails to reflect the manipulation of the other variable. A twodimensional solution can be achieved, but only by directed instructional procedures. Is the two-dimensional solution to equisimilarity of two-dimensional tones the creation of the listener or of the experimenter?

\section{REFERENCES}

GARNER, W. R. The processing of information and structure. New York: Erlbaum-Wiley, 1974.

SHEPARD, R. N. Attention and the metric structure of the stimulus space. Journal of Mathematical Psychology, 1964, 1, 54-87.

ZAGORSKI, M. A. A topological test of metric models of stimulus similarity. Unpublished doctoral dissertation, Department of Psychology, Indiana University, 1973.

(Received for publication January 3, 1979.) 